\title{
Cortical topological network changes following optic neuritis
}

Yael Backner, MSc, Ido Ben-Shalom, BMedSc, Joseph Kuchling, MD, Nadja Siebert, MD, Michael Scheel, MD, Klemens Ruprecht, MD, Alexander Brandt, MD, Friedemann Paul, MD, * and Netta Levin, MD, PhD*

Neurol Neuroimmunol Neuroinflamm 2020;7:e687. doi:10.1212/NXI.0000000000000687

\section{Abstract}

\section{Objective}

To differentiate between visual cortical network topology changes following optic neuritis (ON) stemming from different inflammatory disease types, we used mathematical graph theory-based tools to analyze functional imaging data.

\section{Methods}

Sixty-two patients were recruited into this cross-sectional study, 23 of whom had neuromyelitis optica spectrum disorder (NMOSD) with ON, 18 with clinically isolated syndrome (CIS)-ON, and 21 with other CIS episodes. Twenty-six healthy controls (HCs) were also recruited. All participants underwent resting-state functional MRI. Visual networks were defined using 50 visual regions of interest. Analysis included graph theory metrics, including degree, density, modularity, and local and global efficiency.

\section{Results}

Visual network density shows decreased connectivity in all patient groups compared with controls. A higher degree of connections is seen in both ON groups (CIS and NMOSD) compared with the the non-ON group. This pattern is most pronounced in dorsal-lateral regions. Information transfer efficiency and modularity were reduced in both CIS groups, but not in the NMOSD group, compared with the HC group.

\section{Conclusions}

Visual network density appears affected by the neurologic deficit sustained (ON), and connectivity changes are more evident in dorsal-lateral regions. Efficiency and modularity appear to be associated with the specific disease type (CIS vs NMOSD). Thus, topological cortical changes in the visual system are associated with the type of neurologic deficit within the limits set on them by the underlying pathophysiology. We suggest that cortical patterns of activity should be considered in the outcome of the patients despite the localized nature of $\mathrm{ON}$.

\author{
Correspondence \\ Dr. Levin \\ netta@hadassah.org.il
}




\section{Glossary}

CIS = clinically isolated syndrome; EDSS = Expanded Disability Status Scale; FEF = frontal eye field; FLAIR = fluid-attenuated inversion recovery; HC = healthy control; NMOSD = neuromyelitis optica spectrum disorder; $\mathbf{n O N}=$ nonoptic neuritis; $\mathbf{O N}=$ optic neuritis; ROI = region of interest; RS-fMRI = resting-state functional MRI.

Following an MS episode, recovery involves the resolving of inflammation and remyelination. However, other processes, such as functional cortical network changes, may also have a role to play. ${ }^{1}$ Whether such changes are driven by the specific neurologic deficit experienced by the patient or by the underlying disease pathophysiology and are they specific to certain regions are questions that remain unanswered.

The visual system is a suitable model to address this. Although optic neuritis (ON) is a shared neurologic deficit of both MS and neuromyelitis optica spectrum disorder (NMOSD), 2 distinct CNS inflammatory diseases, in patients with MS, the same underlying pathophysiology may or may not involve the optic nerve. ${ }^{2,3}$

NMOSD and MS differ in their pathophysiologic mechanisms: while in MS, demyelination is the primary damage mechanism; in NMOSD, it is secondary to astrocytopathy, and thus the damage is considered more severe. ${ }^{4}$ Furthermore, as NMOSD is antibody mediated, it is more localized to regions containing the antibody target (aquaporin-4). ${ }^{5}$ These differences in severity and damage distribution suggest that cortical network changes following ON may differ in the 2 disease types.

Moreover, our previous works have emphasized the importance of activity along the visual dorsal stream in patients with $\mathrm{ON}^{6-10}$ but our works on connectivity have used wholenetwork resolution. ${ }^{10,11}$ Therefore, to examine whether there are specific connectivity changes within the visual network, we herein used graph theory methods, ${ }^{12,13}$ focusing on its regional measures to pinpoint which visual regions are more affected.

\section{Methods}

\section{Participants}

Sixty-two patients were prospectively recruited into this crosssectional study at the NeuroCure Clinical Research Center, Charité-Universitätsmedizin Berlin, including 18 patients with $\mathrm{ON}$ in context of clinically isolated syndrome (CIS), 21 patients with CIS with nonvisual episodes (CIS-nonoptic neuritis $[\mathrm{nON}]$ ), and 23 patients with NMOSD and previous ON. Since enrollment in the study, 3 patients with CIS-ON and 5 patients with CIS-nON have been diagnosed with early relapsing-remitting MS according to the 2010 revised McDonald criteria. ${ }^{14}$ Patients with NMOSD met the international consensus diagnostic criteria for NMOSD. ${ }^{15}$ In addition, 26 healthy controls (HCs) were also recruited (table 1). Data from these cohorts have been previously published. ${ }^{10,16,17}$

\section{Standard protocol approvals, registrations, and patient consents}

The study was approved by the Charité-Universitätsmedizin Berlin ethics committee and was conducted in accordance with the Declaration of Helsinki. ${ }^{18}$ Written informed consent was obtained for each participant.

\section{Data acquisition and analysis}

MRI data were acquired on a 3T scanner (Magnetom Trio; Siemens, Erlangen, Germany) using a 12-channel head coil. Anatomic sequences included high-resolution T1-weighted images (MPRAGE sequence, repetition time $[\mathrm{TR}] /$ echo time $[\mathrm{TE}]=1,900 / 3.03 \mathrm{~ms}$, field-of-view [FOV] $256 \times 256 \mathrm{~mm}^{2}$, matrix $256 \times 256,176$ slices, slice thickness $1 \mathrm{~mm}$ ) and fluidattenuated inversion recovery (FLAIR)-weighted images. 3D FLAIR images (3D FLAIR/TE/TR $=6,000 / 2,100 / 388 \mathrm{~ms}$, FOV $240 \times 240 \mathrm{~mm}^{2}$, matrix $240 \times 240,176$ slices, slice thickness $1 \mathrm{~mm}$ ) of all patients were checked and verified for T2 lesions by 3 expert raters under the supervision of a board-certified radiologist. Whole-brain segmentation and quantification of lesions of FLAIR images were performed using ITK-SNAP (itksnap. org). ${ }^{19}$ Further lesion analysis was performed using the mrVista software package (VISTA Lab, Stanford University).

Resting-state functional MRI (RS-fMRI) measurements were obtained with an echo-planar imaging sequence $(\mathrm{TR} / \mathrm{TE}=$ $2,250 / 30 \mathrm{~ms}, \mathrm{FOV}=218 \times 218 \mathrm{~mm}^{2}$, matrix $64 \times 64,37$ slices, slice thickness $3.4 \mathrm{~mm}$, sequence duration 9 minutes). Subjects were instructed to lie still with their eyes closed. Functional imaging data preprocessing and analysis were performed using the BrainVoyager software package, version 20.6 (Brain Innovation). Data underwent slice scan time correction, motion correction, and high-pass temporal filtering by BrainVoyager default parameters to remove drift and improve the signal-tonoise ratio. Functional images were coregistered to the anatomic images using trilinear interpolation and transformed into Montreal Neurological Institute space. Each participant's scan was verified to include the entire brain, with particular attention to the occipital lobe. Each scan was manually checked for quality.

\section{Network construction}

Complex network analysis is based on graph theory, a mathematical approach to networks. Briefly, the method requires definition of brain regions as nodes and the search for connections, or edges, between those nodes. These connections, anatomic or functional, can then be thresholded and binarized into connectivity matrices, representing the existence or lack of connection between any 2 nodes in the network. From these matrices, various measures can be extracted to quantify network topology and organization (figure 1). 
Table 1 Study cohort description

\begin{tabular}{|c|c|c|c|c|}
\hline & $\mathrm{HC}$ & CIS-ON & CIS-nON & NMOSD \\
\hline Subjects (n) & 26 & 18 & 21 & 23 \\
\hline $\operatorname{Sex}(f[m])$ & $22(4)$ & $11(7)$ & $11(10)$ & $20(3)$ \\
\hline Age $(y$, mean \pm SD) & $43.7 \pm 15.7$ & $31.2 \pm 7.7^{\mathrm{a}, \mathrm{b}}$ & $33.4 \pm 8.6^{\mathrm{a}, \mathrm{b}}$ & $46.7 \pm 14.5$ \\
\hline Disease duration (mo; mean \pm SD) & NA & $4.63 \pm 5.15^{a}$ & $5.40 \pm 6.67^{a}$ & $94.17 \pm 95.72$ \\
\hline EDSS score (median; range) & NA & $1.5(0-3.5)^{\mathrm{a}}$ & $1.5(0-4.0)^{\mathrm{a}}$ & $4.0(0-6.5)$ \\
\hline RRMS & NA & 3 & 5 & NA \\
\hline AQP4-ab-positive (n) & NA & NA & NA & 19 \\
\hline Bilateral ON & NA & NA & NA & 4 \\
\hline High-contrast visual acuity & $1.02 \pm 0.32$ & $0.91 \pm 0.34$ & $1.01 \pm 0.34$ & $0.75 \pm 0.47$ \\
\hline $\begin{array}{l}\text { Global lesion volume and number } \\
\left(\mathrm{cm}^{3}, \mathrm{n} \text {; mean } \pm \text { SD) }\right.\end{array}$ & NA & $2.52 \pm 3.12,21.44 \pm 22.66$ & $3.69 \pm 5.53,23.23 \pm 22.35$ & $2.58 \pm 3.94,16.74 \pm 19.37$ \\
\hline
\end{tabular}

Abbreviations: AQP4 = aquaporin-4; CIS = clinically isolated syndrome; EDSS = Expanded Disability Status Scale; $\mathrm{HC}=$ healthy control; NA = not applicable; NMOSD = neuromyelitis optica spectrum disorder; nON = nonoptic neuritis; ON = optic neuritis; RRMS = relapsing-remitting MS

a Significantly different than the NMOSD group, $p<0.01$

${ }^{\mathrm{b}}$ Significantly different than the HC group, $p<0.01$.

\section{Visual network node definition}

As we focus in our studies on the visual system, visual network nodes were defined using the probabilistic atlas of Wang et al., ${ }^{20}$ created in Montreal Neurological Institute space and used in visual system-related studies, ${ }^{21-24}$ which afforded us the level of detail we needed. The constructed networks included 50 regions of interest (ROIs) (25 per hemisphere, 8 ventraltemporal regions, 9 dorsal-lateral regions, and 8 parietal-frontal regions, figure 2A, table e-1A, links.lww.com/NXI/A207), each representing a node. Because of the variety in ROI sizes, wishing to minimize bias of variations in ROI size on estimates of connectivity and signal-to-noise ratio differences, the ROIs were arbitrarily constricted to a set size of $5 \times 5 \times 5$ voxels, positioned at the center of each atlas-defined ROI. The specific size was set so that all constricted regions would be within the borders of their respective original ROIs.

\section{Motor network node definition}

In addition to the visual network, we wished to examine whether similar changes to those in the visual network of patients with ON may also be found in other networks in patients with corresponding CIS episodes. As many of the patients with CIS-nON have experienced motor symptoms, the motor network was also defined, based on the Harvard-Oxford cortical and subcortical structural atlas, included in FMRIB Software Library, ${ }^{25}$ and on several motor network-related studies. ${ }^{26-28}$ The constructed networks included 12 ROIs (6 per hemisphere, figure $2 \mathrm{~B}$, table e-1B, links.lww.com/NXI/ A207). The selected ROIs were pure motor regions to provide us with a clean cutoff between networks and participants and no overlap existed between the 2 networks used. ROIs were constricted to 125 voxels, as in the visual network. Because of the variety of CIS episodes included in the CIS-nON group, for

Figure 1 Visualization of the translation process from atlas to brain network

A

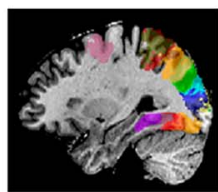

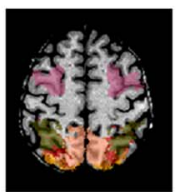

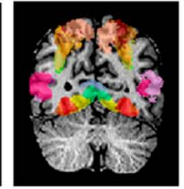

B Left hemisphere

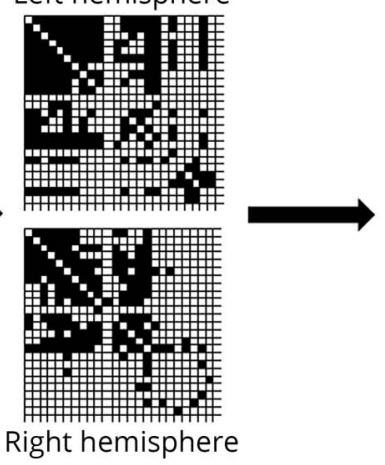

C

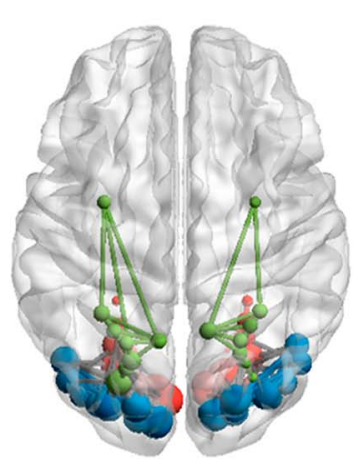

(A) Original Wang et al. ${ }^{20}$ atlas as transformed into BrainVoyager. (B) Creating thresholded and binarized matrices representing all the connections in both hemispheres. (C) A visual network graph representation (healthy control group). 

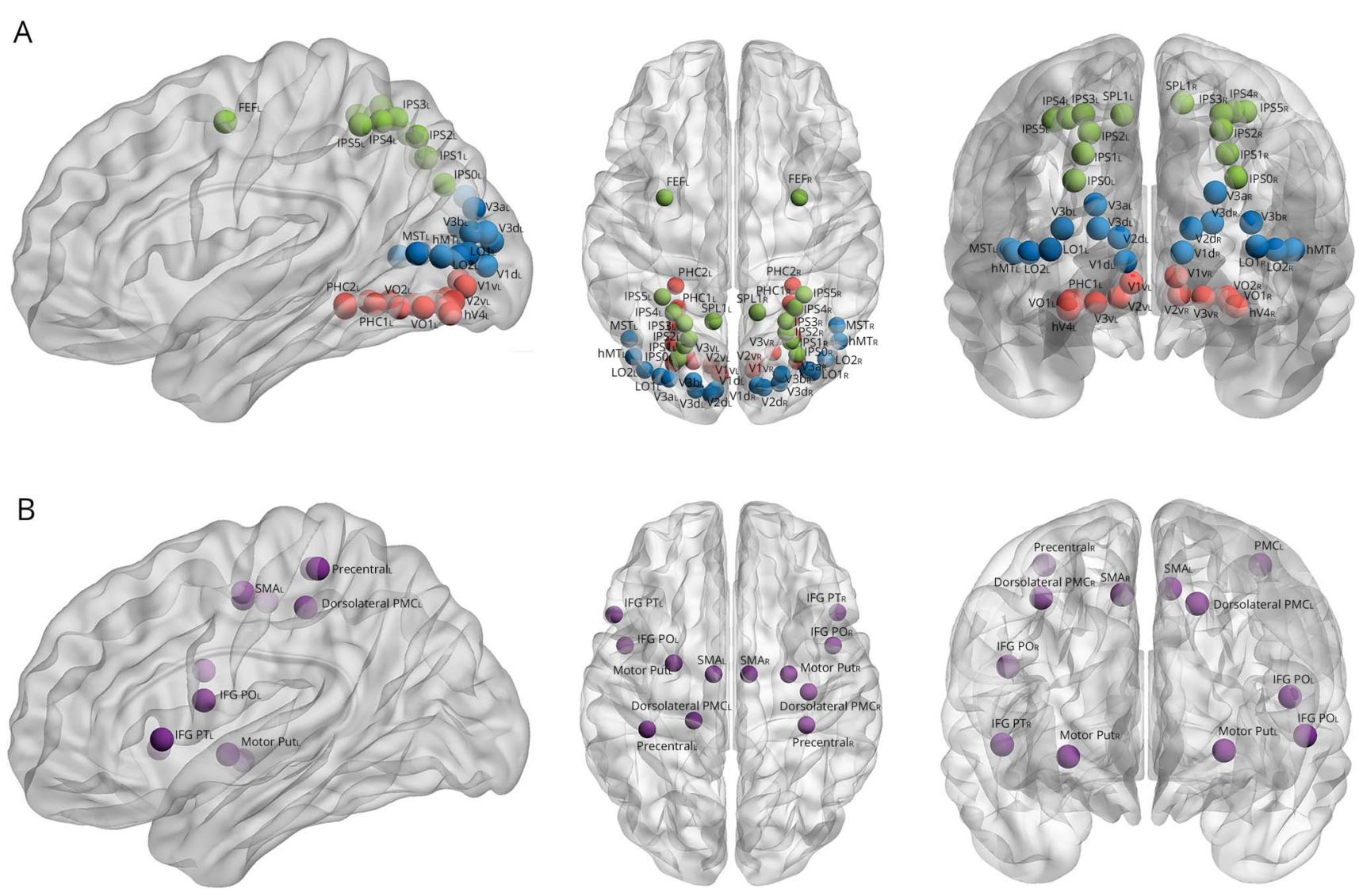

(A) Visual network ROIs, color coded according to classification in Wang et al. ${ }^{20}$-ventral-temporal (red), dorsal-lateral (blue), and parietal-frontal (green). (B) Motor network ROls. Visualization created using Xia M, et al. ${ }^{55} \mathrm{~d}=$ dorsal; FEF = frontal eye field; hMT = human middle temporal region; hV4 = human visual region V4; IFG = inferior frontal gyrus; IPS = intraparietal sulcus; LO = lateral occipital cortex; M1 = primary motor cortex; MST = medial superior temporal area; $\mathrm{PO}=$ pars opercularis; $\mathrm{PT}=$ pars triangularis; $\mathrm{SMA}=$ supplementary motor area; $\mathrm{SPL}=$ superior parietal lobule; $\mathrm{v}=$ ventral; $\mathrm{VO}=$ ventral occipital cortex; $\mathrm{V} 1=$ primary visual cortex; $\mathrm{V} 2$ = secondary visual cortex; V3 = visual area V3.

the motor network analyses, we have further divided the CISnON group into patients with motor symptoms (CIS-nONmotor, $\mathrm{n}=10)$ and with other nonmotor symptoms, mainly sensory (CIS-nON-other, $\mathrm{n}=11$ ).

\section{Matrix preparation}

The average RS-fMRI time course of all voxels within each ROI was extracted, and Pearson correlation coefficients, representing connectivity strength between regions, were calculated between each pair of ROIs in each hemisphere, resulting in two $25 \times 25$ hemispheric visual matrices for each subject and two $6 \times 6$ motor matrices. Each correlation matrix was thresholded and binarized at a range of correlation thresholds (0.1-0.5 for the visual network and $0.1-0.2$ for the motor network, at increments of 0.05 ). The highest correlation threshold was set as the last threshold in which all subjects still had any connection between ROIs. Four patients with CIS-ON and 2 patients with CIS-nON did not have connections in the highest threshold of the motor network. Analyses have been performed with and without these patients, and no significant differences were found.

\section{Network analysis}

Both local and global network properties were studied using the MATLAB Brain Connectivity Toolbox. ${ }^{12}$ Here, we briefly list the network analysis metrics chosen to characterize the networks. All parameters were integrated over the various thresholds used to create a single value for analysis. Graphs presenting the metrics used over the entire range of correlation thresholds are presented in figure e-1 (links.lww.com/ NXI/A207). It should be noted that most metrics chosen could not be used in the motor network because of the small number of regions used and the largely disconnected networks obtained.

\section{Basic network topology analysis}

To characterize the general structure of the network in the local (nodal) level, the nodal degree of the various ROIs was explored. The group mean degree of each given ROI was compared between groups to examine changes following $\mathrm{ON}$ and verify whether any region or subdivision of regions showed greater change. Network density, the proportion of existing connections in the network out of all possible connections, based on the entire network's degree, was also 
calculated and compared to examine the global network differences between groups.

\section{Global network analysis}

To examine the topological organization of the studied networks, 6 global network metrics were explored. Because this study explored only nodes within the visual system, we were interested in seeing whether small-worldness, a ubiquitous trait reported in brain networks, also exists within the visual network. To that end, we included 4 parameters used to calculate small-worldness-clustering coefficient $C_{p}$, harmonic mean of shortest path lengths $L_{p}$, and their normalized counterparts. These were defined as in references 29 and 30. A typical small-world network should exhibit $\gamma>1$ and $\lambda \approx 1$. The harmonic mean was chosen, rather than the more commonly used characteristic path length, because most of the networks in our study are at least partially disconnected and therefore have infinite characteristic path length. ${ }^{31}$

To examine segregation and integration within the visual network, we first looked at the 2 efficiency metrics, the global and local efficiency parameters. Global efficiency is the reciprocal of the harmonic mean, thought to represent how efficiently information is exchanged within the network. Local (or nodal) efficiency is defined for the single node, localizing global efficiency effects to specific nodes, giving a measure of how well that region is integrated within the network. $^{32}$

To further examine segregation and integration, we also calculated modularity. ${ }^{33}$ This measure of network segregation quantifies the degree to which the network may be subdivided into nonoverlapping groups of ROIs, which are densely interconnected within and sparsely connected without.

\section{Statistical analysis}

Data were examined for normality using QQ-plots and Shapiro-Wilk tests. The results variables were non-normally distributed, and thus, for univariate comparisons between groups, we used the Kruskal-Wallis test. To assess the independent effect of group designation on the result variables, after checking compliance with model assumptions, multivariate linear models were built using age, sex, and Expanded Disability Status Scale (EDSS) score as covariates. Statistical significance was assessed at $p<0.05$ level. To control the false discovery rate, multiple comparison correction was performed using the Benjamini-Hochberg method. All calculations were performed using $\mathrm{R}$ version 3.5.1 ( $\mathrm{R}$ Foundation for Statistical Computing, Vienna, Austria).

\section{Data availability}

The data sets generated for this study are available on request from the corresponding author.

\section{Results}

\section{Research cohort characteristics}

A summary of demographic and clinical measures is presented in table 1. Differences were found in age, disease duration, and EDSS score between the 2 CIS groups and the HC and NMOSD groups $(p<0.01$ for all comparisons). Male/female ratios were also different between those groups. Therefore, age, sex, and EDSS score were added as covariates to all analyses and were found to have no effect on the results. Disease duration was not included as a covariate because it was highly correlated with age of patients $(p=0.007)$. Visual acuity did not significantly differ between any of the groups and therefore was not included as covariate, either.

No significant differences were found in lesion number, volume, or specific location in the 3 patient groups. No significant differences were found between hemispheres in any of the metrics. However, significant results between groups were more pronounced in the left hemisphere, and therefore, only these results are presented. For the motor network, significant results were found only in the right hemisphere, and those are the results presented. Results for the unpresented hemispheres can be seen in the supplemental materials (links.lww.com/NXI/A207).

\section{Visual network results}

\section{Small-worldness apparent in visual networks}

By the criteria defined for small-worldness, the healthy visual network appears to display a small-world structure. All the participants in the HC group have shown properties within the criteria $(\lambda$ values were nearly 1 in all participants and thresholds; $\gamma$ values were mostly higher than 1 , barring a few inconsistent exceptions). The same was shown in the patient groups, excluding 1 patient with CIS-ON and 1 patient with CIS-nON who failed to meet the $\gamma>1$ criterion. To verify whether this deviation from criteria somehow influences our group results, analyses were performed with and without these patients, and no significant differences were found, so both were included in the results. The small-world criteria curves for each group are presented in figure e-2 (links.lww.com/NXI/A207).

\section{Basic network topology patterns}

Figure 3 presents a visualization of the visual network for the 4 groups. The normal network displayed by the $\mathrm{HC}$ cohort (figure 3A) appears to be more connected than all 3 patient groups (figure $3, \mathrm{~B}-\mathrm{D}$ ) in all 3 region subdivisions (ventraltemporal in red, dorsal-lateral in blue, and parietal-frontal in green). The NMOSD group (figure 3B) and the CIS-ON group (figure $3 \mathrm{C}$ ) are quite similar in appearance and better connected than the CIS-nON group (figure 3D), which displays the highest disconnection.

This connectivity pattern, according to which: HCs > NMOSD $\sim$ CIS-ON $>$ CIS-nON, is seen globally using the density metric. $\mathrm{HC}$ density $(0.28 \pm 0.09)$ is higher than CIS-nON density (0.19 

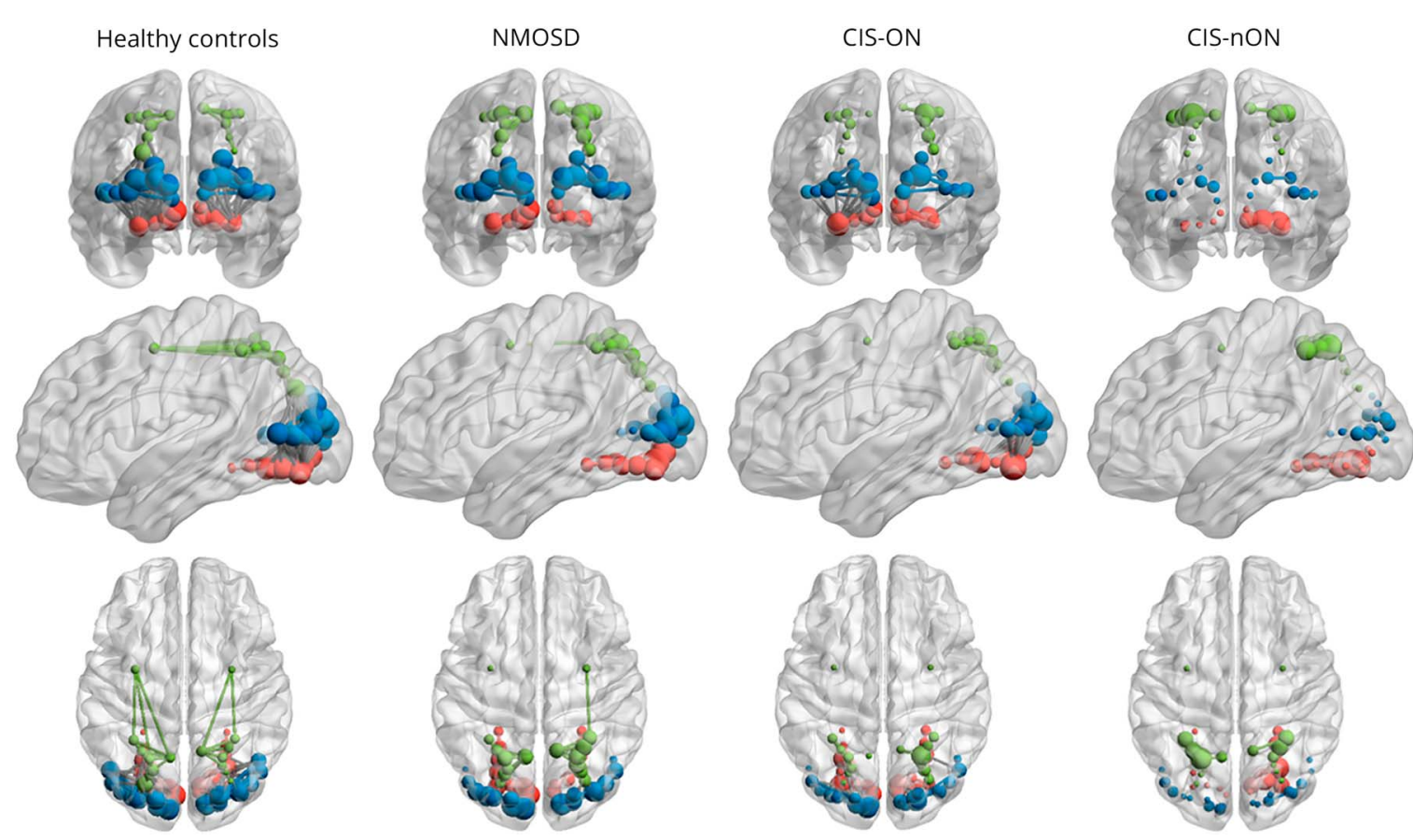

Considered at correlation threshold $r=0.5$, shown in the coronal (back view, top), sagittal (lateral left hemisphere view, middle), and axial (dorsal view, bottom) planes. Nodes (regions of interest) are color coded according to their classification in Wang et al. ${ }^{20}$-ventral-temporal (red), dorsal-lateral (blue), and parietalfrontal (green). The size of nodes reflects the relative degree of a given region in the network. Disconnected regions are presented using an arbitrarily selected size for visualization purposes. Visualization created using reference 55. CIS = clinically isolated syndrome; NMOSD = neuromyelitis optica spectrum disorder; nON = nonoptic neuritis; ON = optic neuritis.

$\pm 0.1, p=0.0009)$, and it is higher, though not significantly so, than the density of the CIS-ON group $(0.22 \pm 0.1, p=0.05)$. The NMOSD group $(0.24 \pm 0.1)$ density was higher than that of the CIS-nON group, but not significantly so $(p=0.06)$. No significant differences were found between the CIS-ON and NMOSD groups or between the NMOSD and HC groups.

The mean degree of the HC group was significantly higher than that of the CIS-nON group in all visual regions, although not all survived multiple comparison correction. Most surviving comparisons were in low-order and dorsal-lateral regions (lower-ventral: V1v and V2v; lower-dorsal: V1d, V2d, and V3d; dorsal-lateral: MST, hMT, and V3b; frontal-parietal: IPSO, IPS1, and IPS2).

In addition, the pattern found using the density metric, by which HCs $>$ NMOSD $\sim$ CIS-ON $>$ CIS-nON, exists mostly in the dorsal-lateral region subdivision. The ventral-temporal subdivision (excluding the very early regions, V1v and V2v) generally shows no significant differences between groups. The parietal-frontal subdivision shows either no significant differences between groups at all (IPS3, IPS4, and IPS5) or a pattern where the 2 CIS groups show no difference between them but lower mean degree than both the HC and NMOSD groups (SPL1, frontal eye field [FEF], $p=0.06$ ). Figure 4 shows an example from each subdivision, displaying the emerging patterns. The average degrees for each ROI can be found in table e-2 (links.lww.com/NXI/A207).

\section{Network segregation and integration patterns}

All segregation and integration metrics reveal connectivity differences unlike those for the basic network topology, showing a pattern by which $\mathrm{HC} \sim \mathrm{NMOSD}>\mathrm{CIS}-\mathrm{ON} \sim$ CIS-nON. This is in contrast to the basic topology pattern (achieved using the degree and density metrics), which highlighted the similarity in topology in different diseases sharing the same neurologic deficit $(\mathrm{HC}>\mathrm{NMOSD} \sim \mathrm{CIS}-\mathrm{ON}>$ CIS-nON).

Modularity results (figure 5A) show differences between the $\mathrm{HC}$ and CIS-nON groups $(0.39 \pm 0.05$ and $0.3 \pm 0.08$, respectively, $p=0.004)$, as well as between the NMOSD $(0.37 \pm$ $0.05)$ and CIS-nON groups $(p=0.016)$, but no significant differences between the HC and NMOSD groups. The modularity result of the CIS-ON group $(0.33 \pm 0.09)$ is not significantly different from any of the other groups but is quantitatively closer to the CIS-nON group.

The harmonized mean of the characteristic path length (figure 5B) shows a difference between the $\mathrm{HC}$ and CIS-nON groups $(0.75 \pm 0.44$ and $2.56 \pm 2.91$, respectively, $p=0.01)$. Although the difference between the CIS-nON and NMOSD groups $(0.83 \pm 0.37, p=0.087)$ is not significant, the NMOSD group displays a shorter path length. The harmonized mean of the CIS-ON group $(2.11 \pm 2.79)$ was not significantly different from any of the groups, and no significant difference was found between the HC and NMOSD groups. 


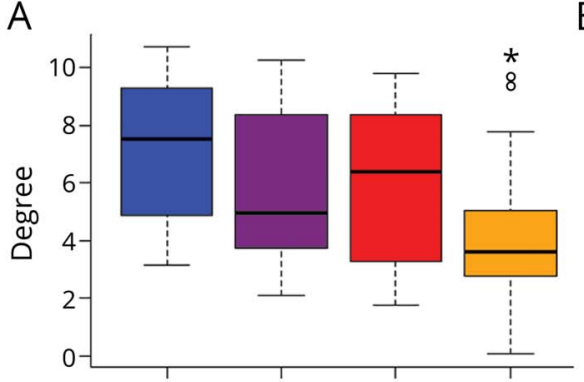

B

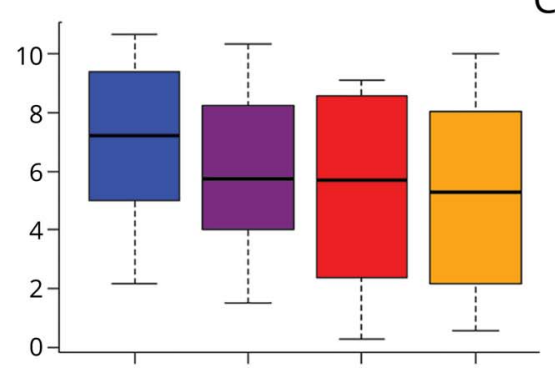

C

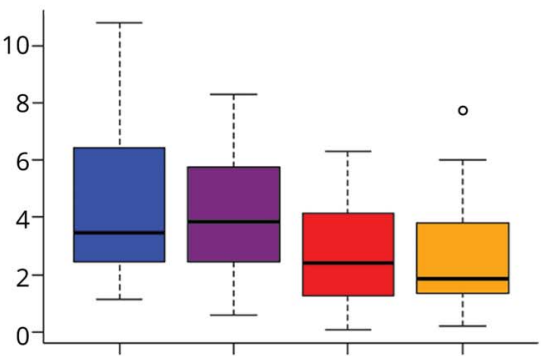

HCS in blue, NMOSD in purple, CIS-ON in red, and CIS-nON in orange. (A) hMT (dorsal-lateral subdivision); (B) VO1 (ventral-temporal subdivision); (C) FEF (frontal eye field, parietal-frontal subdivision). *Significantly different than HC. CIS = clinically isolated syndrome; HC = healthy control; hMT = human middle temporal region; NMOSD = neuromyelitis optica spectrum disorder; nON = nonoptic neuritis; ON = optic neuritis.

Similarly, global efficiency (figure 5C) shows change between the $\mathrm{HC}$ and CIS-nON groups ( 0.35 and 0.25 , respectively, $p=$ 0.008). Again, no significant difference was found between the NMOSD (0.31) and CIS-nON groups $(p=0.093)$, but the NMOSD group quantitatively shows higher efficiency. The CIS-ON group once again shows no significant differences (0.28) from any group, but is between the NMOSD and CIS-nON groups in terms of global efficiency.

Local efficiency showed similar results with differences found between the CIS-nON group and the HC and NMOSD groups, though mostly in high-order regions, particularly in the dorsal-lateral and parietal-frontal region subdivisions (lowerventral: V1v, CIS-nON vs HCs only; lower-dorsal: V3d; dorsallateral: MST, hMT, V3a CIS-nON vs HCs only, LO1, LO2, V3b CIS-nON vs HCs and NMOSD; frontal-parietal: IPS3, IPS4, IPS5, SPL1 vs HCs only, IPS2, FEF vs HCs and NMOSD; regions V3b, IPS3, IPS5 have also shown significant differences between HCs and CIS-ON). Details on local efficiency for each ROI can be seen in table e-3 (links.lww.com/ NXI/A207).

\section{Motor network results}

Differences between groups were found using the degree metric in the motor putamen, between the HC group, which was most connected (0.54), and all patient groups (CIS-ON, 0.28; CIS-nON-motor, 0.225; CIS-nON-other 0.28; $p<0.01$ for all) except for the NMOSD group $(0.38 ; p=0.1$ after multiple comparison correction). No significant differences were found between any patient groups.

In the pars opercularis (inferior frontal gyrus-pars opercularis), differences were found between the CIS-nON-motor group $(0.25)$ and the HC $(0.57 ; p=0.004)$ and NMOSD (0.49; $p=0.03)$ groups. Density and global efficiency metrics also show differences between the HC group and the CIS-nON-

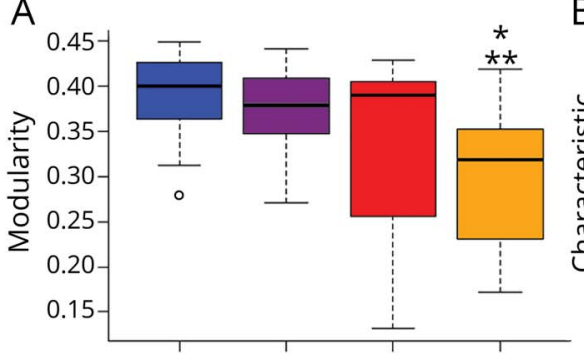

B

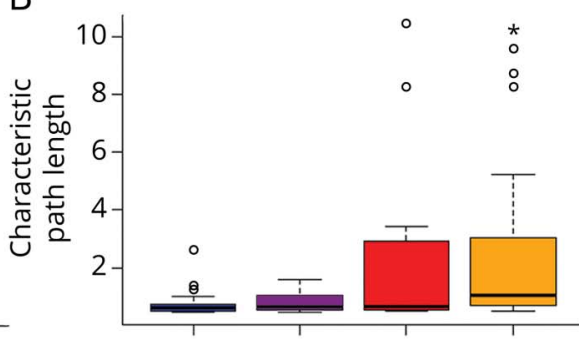

C

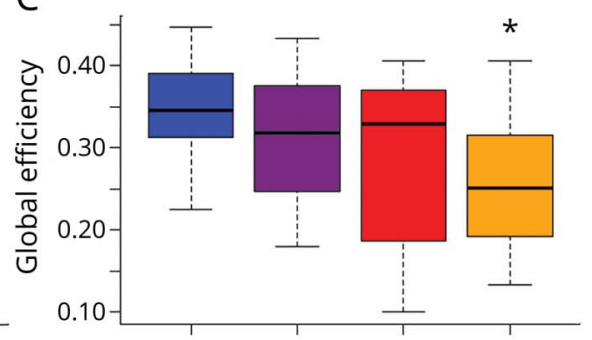

HCs in blue, NMOSD in purple, CIS-ON in red, and CIS-nON in orange. (A) Modularity; (B) harmonized mean of characteristic path length; (C) global efficiency. *Significantly different than HC; **Significantly different than NMOSD. CIS = clinically isolated syndrome; HC = healthy control; NMOSD = neuromyelitis optica spectrum disorder; nON = nonoptic neuritis; $\mathrm{ON}=$ optic neuritis. 
motor group (density: HCs, 0.12; CIS-nON-motor, 0.07; $p=$ 0.005; efficiency: HCs, 0.14; CIS-nON-motor, 0.09 ; $p=0.001$ ). Density also shows difference between the $\mathrm{HC}$ group and the CIS-ON group $(0.11 ; p=0.04)$. Other comparisons did not survive correction, but surprisingly the general pattern remains here, similarly to the visual network: HCs > NMOSD > CIS$\mathrm{ON} \sim \mathrm{CIS}$-nON-other $>$ CIS-nON-motor. Results are presented in figure e-3 (links.lww.com/NXI/A207).

\section{Discussion}

In this study, we addressed visual cortical network patterns in inflammatory diseases following $\mathrm{ON}$. To that end, we included 2 prototypic autoimmune CNS diseases with distinct underlying pathophysiology, MS and NMOSD. ${ }^{34,35}$ These diseases both share visual dysfunction caused by $\mathrm{ON}$ as clinical commonality. Through these 2 disease types, we have attempted to discover whether changes to the visual system following $\mathrm{ON}$ are driven just by the specific neurologic visual deficit or also by the underlying pathophysiology. We used graph analysis methods to investigate topological and local changes in resting-state visual networks of patients in those 2 disease groups, further dividing the MS group into patients with and without $\mathrm{ON}$.

Our main findings showed 2 distinct patterns of change in the visual networks of the patient groups. First, the highly connected normal visual network is affected in all 3 disease groups, showing reduced density and changes in topology. These differences from HCs, however, are most pronounced in the CIS-nON group. The 2 ON groups (NMOSD and CIS$\mathrm{ON}$ ) showed remarkably similar results in density and degree distribution, particularly in lower and dorsal-lateral regions, both higher than those of patients with CIS-nON. This suggests changes unique to the directly affected system (visual input). We cautiously suggest that these changes may reflect an attempt to compensate for the damage incurred, lasting even when the underlying pathology has existed for years, considering the relatively long disease durations displayed by the NMOSD compared with the CIS-ON group.

Second, measures of network segregation and integration, defining efficiency of information transfer and local structure, showed similarity between the MS-background groups (CISON and CIS-nON), whereas the NMOSD group showed results closer to those of the HCs. This suggests that network segregation and integration may be more affected by type of underlying pathophysiology, rather than the specific neurologic deficit.

The question of whether cortical reorganization plays a role in recovery from $\mathrm{ON}$ has long been discussed. Early studies focused mainly on cortical activity patterns in response to visual tasks presented to the affected eye. Some studies have suggested that adaptive cortical plasticity following $\mathrm{ON}$ involves increased activity in higher visual areas, assisting in overcoming visual insult. ${ }^{36-39}$ However, later studies suggested that cortical activation reflects the visual percept rather than demonstrates plasticity. ${ }^{8}$ In recent years, the importance of considering cortical networks rather than regional activity has emerged. Functional connectivity, assessing the synchronized fluctuations in the fMRI signal between regions, has shown sensitivity to neurologic damage and so it was suggested that connectivity rather than activity may play a role in clinical outcome. However, as this is a cross-sectional study, and no clinical measures were included, this remains hypothetical.

In MS and NMOSD, much emphasis has been given to changes in functional connectivity in sensorimotor networks. Functional connectivity within the motor network was found to reflect motor function ${ }^{40}$ and to change in response to rehabilitative intervention ${ }^{41}$ in patients with MS. Whether such changes reflect compensatory mechanisms or loss of function is still a matter of debate. ${ }^{42,43}$ It has been suggested that the interpretation of such observations depends on lesion load and disease duration. ${ }^{44}$

Our motor network results show no compensatory changes in the subgroup of nON patients with motor symptoms. On the contrary, specific clinical motor involvement resulted in reduced connectivity within the motor cortex. These results are particularly prominent in view of the opposite results obtained in the visual network, where the relevant neurologic deficit seemed to be associated with elevated connectivity. We suggest that this may be the result of lesions located close to motor regions compared with the more peripheral damage sustained through ON, causing greater direct damage to the system. Another piece of evidence supporting this suggestion is the denser motor network seen in the NMOSD group, who almost all had myelitis in addition to ON (19 of 23 patients). This finding may be the motor network's equivalent of response to distant damage. However, because of the small numbers of patients included in the nonmotor subgroup and of patients with nonmyelitis NMOSD, these speculations should be taken with caution.

Although much attention has been given to the motor networks in recent years, the visual system has received less attention, usually as a small part of a wider study. ${ }^{43,45}$ A study focusing on the visual system of patients with NMOSD and previous $\mathrm{ON}$ has shown increase in the functional connectivity of the primary visual network compared with those without ON. ${ }^{11}$ These findings were also associated with greater retinal damage and reduced visual acuity, suggesting an attempt at cortical reorganization. Similar findings were seen in our previous work, showing an increase in visual network connectivity in patients with CIS-ON compared with CIS-nON. ${ }^{10}$ A MEG/ OCT study of the visual system in patients with MS has succeeded in differentiating between patients with and without $\mathrm{ON}$ based on a unique pattern of connectivity that specifically involves the visual system. ${ }^{46}$ These studies mostly focus on the neurologic deficit as the driving force behind the effects observed in MS and NMOSD connectivity and are congruent 
with the general connectivity patterns presented in our current study.

However, another cause for connectivity change may be the specific type of disease. Very few studies have tried to compare connectivity between these 2 similar, yet dissimilar, disease groups. Of these, one has studied the functional and structural connectivity of the thalamus, finding structural changes in both diseases but functional disconnection only in MS. ${ }^{47}$ Another study used a wide array of tests to differentiate between the 2 disease types, showing that the top 2 modalities useful in this were white matter lesion load and functional connectivity. ${ }^{48}$ In our results, the white matter lesion element appears negligible because both groups exhibit similar lesion number and volume, and even lesion distribution was not localized in any of the groups. And yet, the segregation and integration patterns of the functional visual network differed between MS and NMOSD, with those of the latter more closely resembling that of HCs. We suggest that this marked difference may stem from differences in normal-appearing white matter. Diffusion tensor imaging studies have shown diffusion abnormalities in both disease types, but patients with MS display widespread and more severe degree of abnormality. ${ }^{49-51}$ This may explain the difference in the segregation and integration properties of functional connectivity between the 2 groups.

The use of graph-based analysis has enabled us to explore both these driving forces of connectivity alterations, the specific neurologic deficit and the disease type itself, that appear to be associated with different types of change. Another advantage of this method is its ability to examine connectivity not only in a holistic manner. In a work from 2015, the advantage of graph-based analysis in MS was presented. Although emphasis was on cognitive changes, it was suggested that graph theory should be used to overcome the simplistic and incomplete interpretations of previous methods. ${ }^{52}$ Work on functional connectivity of the visual network in MS over the years has mainly examined the network as a single entity, using independent component analysis ${ }^{10,53}$ or seed-region analysis. ${ }^{54}$ However, the introduction of graph theory methods ${ }^{12}$ has opened new possibilities in studying the affected network. In MS, the different works report both decreased global connectivity attributed to disease course and adaptation patterns with increased local connectivity and modularity. ${ }^{13}$ Our current findings, highlighting reorganization in dorsal-lateral regions, known to be involved in dynamic perception processes, are consistent with our previous works that emphasized persistent motion perception impairment following ON.,8 Based on our previous works in the field, we suggest that the dorsal stream-specific increase in connectivity in our current study may be part of a recovery process involving functional modifications. Because both ON groups exhibit such a pattern, this supports the notion that the neurologic deficit elicits these changes. As this is a cross-sectional study, this remains only a suggestion.
This study has several limitations. First, graph theory methods are often used on larger subject groups. However, the significance of the results and their consistency with our previous findings convince us that our current findings are true. Second, the differences in age in all groups and in disease duration between the disease groups are problematic, but these measures were factored into the various analyses and had no significant influence on the results. Third, our assumptions regarding the existence of compensatory mechanisms are based on the patterns emerging and former findings of our research. Further light could have been shed on the matter, had behavioral measures been included, as well as a longitudinal design. It should be further noted that this study analyzed only functional connections within the visual system, without the added information that could have been gleaned from the complementing structural connectome, limiting the ability to disentangle compensatory changes from structural damage. Furthermore, although the differences between groups may indeed be interpreted as reorganizational changes of the visual network in response to damage, they may also reflect differential level of damage between individuals. This issue should be further investigated using a different study design because it could not be covered under the scope of the current study.

To conclude, the visual network, highly connected in HCs, is made less connected by disease, be it MS or NMOSD. However, a higher degree of connections was observed in the ON groups (CIS-ON and NMOSD) compared with the $\mathrm{nON}$ group. This suggests that the system responds to specific damage caused to the anterior visual pathway, bringing connectivity closer to that of a normal network, regardless of disease type. On the other hand, network segregation and integration and the efficiency of information transfer appear more affected by disease type, altered in the MS groups, but not in NMOSD, particularly in higher regions. Therefore, we suggest that the response to damage is dependent on the type of neurologic deficit within the limitations of the underlying pathophysiology.

\section{Study funding}

Supported by the National Multiple Sclerosis Society (research grant RG-1802-30165) and the Applebaum Foundation. F. Paul was supported by Deutsche Forschungsgemeinschaft (DFG Exc 257).

\section{Disclosure}

Y. Backner, I. Ben-Shalom, J. Kuchling, N. Siebert, M. Scheel, K. Ruprecht, A. Brandt, F. Paul, and N. Levin report no disclosures. Go to Neurology.org/NN for full disclosures.

\section{Publication history}

Received by Neurology: Neuroimmunology \& Neuroinflammation October 11, 2019. Accepted in final form January 10, 2020. 
Appendix Authors

\begin{tabular}{|c|c|c|}
\hline Name & Location & Contribution \\
\hline $\begin{array}{l}\text { Yael } \\
\text { Backner, } \\
\text { MSc }\end{array}$ & $\begin{array}{l}\text { Hadassah-Hebrew } \\
\text { University Medical } \\
\text { Center, Jerusalem, } \\
\text { Israel }\end{array}$ & $\begin{array}{l}\text { Designed and } \\
\text { conceptualized the study; } \\
\text { analyzed the data; drafted } \\
\text { the figures; and drafted } \\
\text { the manuscript for } \\
\text { intellectual content }\end{array}$ \\
\hline $\begin{array}{l}\text { Ido Ben- } \\
\text { Shalom, } \\
\text { BMedSc }\end{array}$ & $\begin{array}{l}\text { Hadassah-Hebrew } \\
\text { University Medical } \\
\text { Center, Jerusalem, } \\
\text { Israel }\end{array}$ & $\begin{array}{l}\text { Analyzed the motor } \\
\text { network data }\end{array}$ \\
\hline $\begin{array}{l}\text { Joseph } \\
\text { Kuchling, } \\
\text { MD }\end{array}$ & $\begin{array}{l}\text { Charité-Universitätsmedizin } \\
\text { Berlin, Germany }\end{array}$ & $\begin{array}{l}\text { Major role in data } \\
\text { acquisition and revised the } \\
\text { manuscript for intellectual } \\
\text { content }\end{array}$ \\
\hline $\begin{array}{l}\text { Nadja } \\
\text { Siebert, MD }\end{array}$ & $\begin{array}{l}\text { Charité-Universitätsmedizin } \\
\text { Berlin, Germany }\end{array}$ & $\begin{array}{l}\text { Major role in data } \\
\text { acquisition }\end{array}$ \\
\hline $\begin{array}{l}\text { Michael } \\
\text { Scheel, MD }\end{array}$ & $\begin{array}{l}\text { Charité-Universitätsmedizin } \\
\text { Berlin, Germany }\end{array}$ & $\begin{array}{l}\text { Major role in data } \\
\text { acquisition }\end{array}$ \\
\hline $\begin{array}{l}\text { Klemens } \\
\text { Ruprecht, } \\
\text { MD }\end{array}$ & $\begin{array}{l}\text { Charité-Universitätsmedizin } \\
\text { Berlin, Germany }\end{array}$ & $\begin{array}{l}\text { Major role in data } \\
\text { acquisition and revised the } \\
\text { manuscript for intellectual } \\
\text { content }\end{array}$ \\
\hline $\begin{array}{l}\text { Alexander } \\
\text { Brandt, MD }\end{array}$ & $\begin{array}{l}\text { Charité-Universitätsmedizin } \\
\text { Berlin, Germany; University } \\
\text { of California }\end{array}$ & $\begin{array}{l}\text { Major role in data } \\
\text { acquisition }\end{array}$ \\
\hline $\begin{array}{l}\text { Friedemann } \\
\text { Paul, MD }\end{array}$ & $\begin{array}{l}\text { Charité-Universitätsmedizin } \\
\text { Berlin, Germany }\end{array}$ & $\begin{array}{l}\text { Supervised the study and } \\
\text { revised the manuscript for } \\
\text { intellectual content }\end{array}$ \\
\hline $\begin{array}{l}\text { Netta Levin, } \\
\text { MD, PhD }\end{array}$ & $\begin{array}{l}\text { Hadassah-Hebrew } \\
\text { University Medical Center, } \\
\text { Jerusalem, Israel }\end{array}$ & $\begin{array}{l}\text { Conceptualized and } \\
\text { designed the study; } \\
\text { supervised the study; } \\
\text { and drafted the } \\
\text { manuscript for } \\
\text { intellectual content }\end{array}$ \\
\hline
\end{tabular}

\section{References}

1. Schoonheim MM, Filippi M. Functional plasticity in MS Friend or foe? Neurology 2012;79:1418-1419.

2. Costello F. Vision disturbances in multiple sclerosis. Semin Neurol 2016;36:185-195.

3. Abel A, McClelland C, Lee MS. Critical review: typical and atypical optic neuritis. Surv Ophthalmol 2019;64:770-779.

4. Chen JJ, Pittock SJ, Flanagan EP, Lennon VA, Bhatti MT. Optic neuritis in the era of biomarkers. Surv Ophthalmol 2020;65:12-17.

5. Lennon VA, Wingerchuk DM, Kryzer TJ, et al. A serum autoantibody marker of neuromyelitis optica: distinction from multiple sclerosis. Lancet 2004;364:2106-2112.

6. Raz N, Chokron S, Ben-Hur T, Levin N. Temporal reorganization to overcome monocular demyelination. Neurology 2013;81:702-709.

7. Raz N, Dotan S, Chokron S, Ben-Hur T, Levin N. Demyelination affects temporal aspects of perception: an optic neuritis study. Ann Neurol 2012;71:531-538.

8. Raz N, Dotan S, Benoliel T, Chokron S, Ben-Hur T, Levin N. Sustained motion perception deficit following optic neuritis Behavioral and cortical evidence. Neurology 2011;76:2103-2111.

9. Benoliel T, Raz N, Ben-Hur T, Levin N. Cortical functional modifications following optic neuritis. Mult Scler 2017;23:220-227.

10. Backner Y, Kuchling J, Massarwa S, et al. Anatomical wiring and functional networking changes in the visual system following optic neuritis. JAMA Neurol 2018;75:287-295.

11. Finke C, Zimmermann H, Pache F, et al. Association of visual impairment in neuromyelitis optica spectrum disorder with visual network reorganization. JAMA Neurol 2018;75:296-303.

12. Rubinov M, Sporns O. Complex network measures of brain connectivity: uses and interpretations. Neuroimage 2010;52:1059-1069.

13. Fleischer V, Radetz A, Ciolac D, et al. Graph theoretical framework of brain networks in multiple sclerosis: a review of concepts. Neuroscience 2019;403:35-53.

14. Polman CH, Reingold SC, Banwell B, et al. Diagnostic criteria for multiple sclerosis: 2010 revisions to the McDonald criteria. Ann Neurol 2011;69:292-302.

15. Wingerchuk DM, Banwell B, Bennett JL, et al. International consensus diagnostic criteria for neuromyelitis optica spectrum disorders. Neurology 2015;85:177-189.
16. Kuchling J, Backner Y, Oertel FC, et al. Comparison of probabilistic tractography and tract-based spatial statistics for assessing optic radiation damage in patients with autoimmune inflammatory disorders of the central nervous system. Neuroimage Clin 2018; 19:538-550.

17. Oertel FC, Kuchling J, Zimmermann H, et al. Microstructural visual system changes in AQP4-antibody-seropositive NMOSD. Neurol Neuroimmunol Neuroinflamm 2017; 4:e334. doi: 10.1212/NXI.0000000000000334.

18. Association WM. World Medical Association Declaration of Helsinki: ethical principles for medical research involving human subjects. JAMA 2013;310:2191.

19. Yushkevich PA, Piven J, Hazlett HC, et al. User-guided 3D active contour segmentation of anatomical structures: significantly improved efficiency and reliability. Neuroimage 2006;31:1116-1128.

20. Wang L, Mruczek RE, Arcaro MJ, Kastner S. Probabilistic maps of visual topography in human cortex. Cereb Cortex 2014;25:3911-3931.

21. Hindy NC, Ng FY, Turk-Browne NB. Linking pattern completion in the hippocampus to predictive coding in visual cortex. Nat Neurosci 2016;19:665.

22. Cichy RM, Khosla A, Pantazis D, Torralba A, Oliva A. Comparison of deep neural networks to spatio-temporal cortical dynamics of human visual object recognition reveals hierarchical correspondence. Sci Rep 2016;6:27755.

23. Cichy RM, Pantazis D, Oliva A. Similarity-based fusion of MEG and fMRI reveals spatio-temporal dynamics in human cortex during visual object recognition. Cereb Cortex 2016;26:3563-3579.

24. Mackey WE, Winawer J, Curtis CE. Visual field map clusters in human frontoparietal cortex. Elife 2017;6:e22974.

25. Smith SM, Jenkinson M, Woolrich MW, et al. Advances in functional and structura MR image analysis and implementation as FSL. Neuroimage 2004;23:S208-S219.

26. Pool E-M, Rehme AK, Eickhoff SB, Fink GR, Grefkes C. Functional resting-state connectivity of the human motor network: differences between right-and left-handers. Neuroimage 2015;109:298-306.

27. Hardwick RM, Rottschy C, Miall RC, Eickhoff SB. A quantitative meta-analysis and review of motor learning in the human brain. Neuroimage 2013;67:283-297.

28. Rocca MA, Meani A, Riccitelli GC, et al. Abnormal adaptation over time of motor network recruitment in multiple sclerosis patients with fatigue. Mult Scler J 2016;22: 1144-1153.

29. Watts DJ, Strogatz SH. Collective dynamics of "small-world" networks. Nature 1998; 393:440-442.

30. Maslov S, Sneppen K. Specificity and stability in topology of protein networks. Science 2002;296:910-913.

31. Newman ME. The structure and function of complex networks. SIAM Rev 2003;45: 167-256.

32. Latora V, Marchiori M. Efficient behavior of small-world networks. Phys Rev Lett 2001;87:198701

33. Newman ME. Modularity and community structure in networks. Proc Natl Acad Sci USA 2006; 103:8577-8582.

34. Zamvil SS, Slavin AJ. Does MOG Ig-positive AQP4-seronegative opticospinal inflammatory disease justify a diagnosis of NMO spectrum disorder? Neurol Neuroimmunol Neuroinflamm 2015;2:e62. doi: 10 $1212 /$ NXI.0000000000000062.

35. Zekeridou A, Lennon VA. Aquaporin-4 autoimmunity. Neurol Neuroimmunol Neuroinflamm 2015;2:e110. doi: 10.1212/NXI.0000000000000110.

36. Toosy AT, Hickman SJ, Miszkiel KA, et al. Adaptive cortical plasticity in higher visual areas after acute optic neuritis. Ann Neurol 2005;57:622-633.

37. Jenkins TM, Toosy AT, Ciccarelli O, et al. Neuroplasticity predicts outcome of optic neuritis independent of tissue damage. Ann Neurol 2010;67:99-113.

38. Werring DJ, Bullmore ET, Toosy AT, et al. Recovery from optic neuritis is associated with a change in the distribution of cerebral response to visual stimulation: a functional magnetic resonance imaging study. J Neurol Neurosurg Psychiatry 2000;68: 441-449.

39. Levin N, Orlov T, Dotan S, Zohary E. Normal and abnormal fMRI activation patterns in the visual cortex after recovery from optic neuritis. Neuroimage 2006;33 $1161-1168$.

40. Cordani C, Meani A, Esposito F, et al. Imaging correlates of hand motor performance in multiple sclerosis: a multiparametric structural and functional MRI study. Mult Scler 2019:1352458518822145

41. Fling BW, Martini DN, Zeeboer E, Hildebrand A, Cameron M. Neuroplasticity of the sensorimotor neural network associated with walking aid training in people with multiple sclerosis. Mult Scler Relat Disord 2019;31:1-4.

42. Tommasin S, De Giglio L, Ruggieri S, et al. Relation between functional connectivity and disability in multiple sclerosis: a non-linear model. J Neurol 2018;265 2881-2892.

43. Castellazzi G, Debernard L, Melzer TR, et al. Functional connectivity alterations reveal complex mechanisms based on clinical and radiological status in mild relapsing remitting multiple sclerosis. Front Neurol 2018;9:690.

44. Tewarie P, Steenwijk MD, Brookes MJ, et al. Explaining the heterogeneity of functional connectivity findings in multiple sclerosis: an empirically informed modeling study. Hum Brain Mapp 2018;39:2541-2548.

45. Rocca MA, Savoldi F, Valsasina P, et al. Cross-modal plasticity among sensory networks in neuromyelitis optica spectrum disorders. Mult Scler 2019;25:968-979.

46. Tewarie P, Balk LJ, Hillebrand A, et al. Structure-function relationships in the visua system in multiple sclerosis: an MEG and OCT study. Ann Clin Transl Neurol 2017; 4:614-621.

47. Liu Y, Duan Y, Huang J, et al. Multimodal quantitative MR imaging of the thalamus in multiple sclerosis and neuromyelitis optica. Radiology 2015;277:784-792. 
48. Eshaghi A, Riyahi-Alam S, Saeedi R, et al. Classification algorithms with multi-modal data fusion could accurately distinguish neuromyelitis optica from multiple sclerosis. Neuroimage Clin 2015;7:306-314.

49. Kim SH, Kwak K, Hyun JW, et al. Diffusion tensor imaging of normal-appearing white matter in patients with neuromyelitis optica spectrum disorder and multiple sclerosis. Eur J Neurol 2017;24:966-973.

50. Matthews L, Kolind S, Brazier A, et al. Imaging surrogates of disease activity in neuromyelitis optica allow distinction from multiple sclerosis. PLoS One 2015;10:e0137715.

51. Pache F, Zimmermann H, Finke C, et al. Brain parenchymal damage in neuromyelitis optica spectrum disorder-a multimodal MRI study. Eur Radiol 2016;26:4413-4422.
52. Schoonheim MM, Meijer KA, Geurts JJ. Network collapse and cognitive impairment in multiple sclerosis. Front Neurol 2015;6:82.

53. Gallo A, Esposito F, Sacco R, et al. Visual resting-state network in relapsingremitting MS with and without previous optic neuritis. Neurology 2012;79: 1458-1465.

54. Rocca MA, Valsasina P, Leavitt VM, et al. Functional network connectivity abnormalities in multiple sclerosis: correlations with disability and cognitive impairment. Mult Scler 2018;24:459-471.

55. Xia M, Wang J, He Y. BrainNet Viewer: a network visualization tool for human brain connectomics. PLoS One 2013;8:e68910. 


\title{
Neurology \\ Neuroimmunology \& Neuroinflammation
}

\author{
Cortical topological network changes following optic neuritis \\ Yael Backner, Ido Ben-Shalom, Joseph Kuchling, et al. \\ Neurol Neuroimmunol Neuroinflamm 2020;7; \\ DOI 10.1212/NXI.0000000000000687
}

This information is current as of March 2, 2020

\section{Updated Information \& Services \\ References \\ Subspecialty Collections}

Permissions \& Licensing

Reprints including high resolution figures, can be found at:

http://nn.neurology.org/content/7/3/e687.full.html

This article cites 54 articles, 6 of which you can access for free at: http://nn.neurology.org/content/7/3/e687.full.html\#\#ref-list-1

This article, along with others on similar topics, appears in the following collection(s):

Devic's syndrome

http://nn.neurology.org//cgi/collection/devics_syndrome

fMRI

http://nn.neurology.org//cgi/collection/fmri

Multiple sclerosis

http://nn.neurology.org//cgi/collection/multiple_sclerosis

Optic neuritis; see Neuro-ophthalmology/Optic Nerve

http://nn.neurology.org//cgi/collection/optic_neuritis

Plasticity

http://nn.neurology.org//cgi/collection/plasticity

Information about reproducing this article in parts (figures,tables) or in its entirety can be found online at:

http://nn.neurology.org/misc/about.xhtml\#permissions

Information about ordering reprints can be found online:

http://nn.neurology.org/misc/addir.xhtml\#reprintsus

Neurol Neuroimmunol Neuroinflamm is an official journal of the American Academy of Neurology.

Published since April 2014, it is an open-access, online-only, continuous publication journal. Copyright

Copyright (C) 2020 The Author(s). Published by Wolters Kluwer Health, Inc. on behalf of the American

Academy of Neurology.. All rights reserved. Online ISSN: 2332-7812.

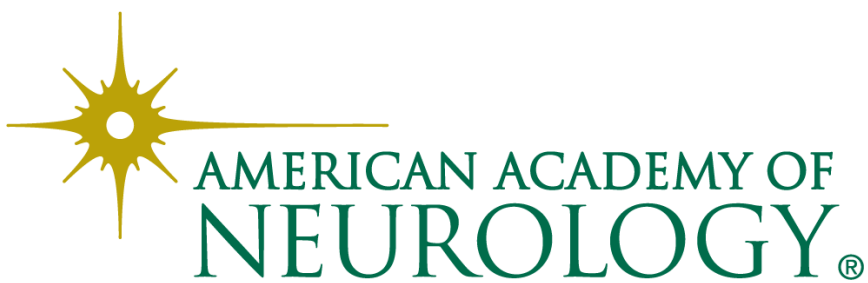

